\title{
Readying CERN for connected device era
}

\author{
Rodrigo Sierra ${ }^{1, *}$, Hubert Odziemczyk ${ }^{1}$ \\ ${ }^{1}$ CERN, IT Department, 1217 Meyrin, Switzerland
}

\begin{abstract}
Whether you consider "IoT" as a real thing or a buzzword, there's no doubt that connected devices, data analysis and automation are transforming industry. CERN is no exception: a network of LoRa-based radiation monitors has recently been deployed and there is a growing interest in the advantages connected devices could bring - to accelerator operations just as much as to building management. Connected devices bring risks as well as advantages, however, and the last thing any business needs is an unsafe, uncoordinated and unmanaged sensor environment. To support the deployment of the LoRa-based radiation sensors, CERN has established both a Low Power Wireless Area Network (LPWAN) to complement the existing wired and wireless networks and a service infrastructure to manage the provisioning, orchestration, data transfer, security and operation for connected devices.
\end{abstract}

\section{Introduction}

The interest in the Internet of Things (IoT) is growing exponentially so multiple technologies and solutions have emerged to connect mostly everything. A 'thing' can be a car, a thermometer or a robot that, when equipped with a transceiver, will exchange information over the internet with a defined service. Therefore, IoT comprises a wide variety of user cases with very different requirements.

IoT is not new; you can think about all random devices connected to the internet since years via Ethernet, Wi-Fi or cellular networks. However, new demands and use cases keep appearing that push the industry to develop new solutions and open new markets.

CERN needed a solution to measure radiation in areas where there was no electricity nor cabling available so the IT department decided to offer a service to support this new requirement.

\section{2 loT Networking}

There are several hundred IoT standards (from connectivity to application level) ${ }^{[1]}$ so we could consider several parameters to choose the right network according to the user requirements:

\footnotetext{
* Corresponding author: rodrigo.sierra@,cern.ch
} 
- Data rate: bps to Gbps.- Traffic pattern:

- Node to network (e.g., alarms, sensors).

- Network to node (e.g., control, actuators). - Bidirectional communication.

- Cabled or wireless.

- Covered area: some meters around the emitter, a room, a building, a campus, a country....

- Range covered by a single transmitter: from centimetres to kilometres.

- Power:

- Do you have a power supply or have to run on batteries?

- How long your batteries should last?

- Mobility: static or mobile, moving speed...

- Scalability.

- Security.

- Price.

- Available Spectrum: licensed vs. Unlicensed.

If we concentrate on the wireless networks; we could make a first simplification according to the bandwidth requirements and the range over which communications are needed.

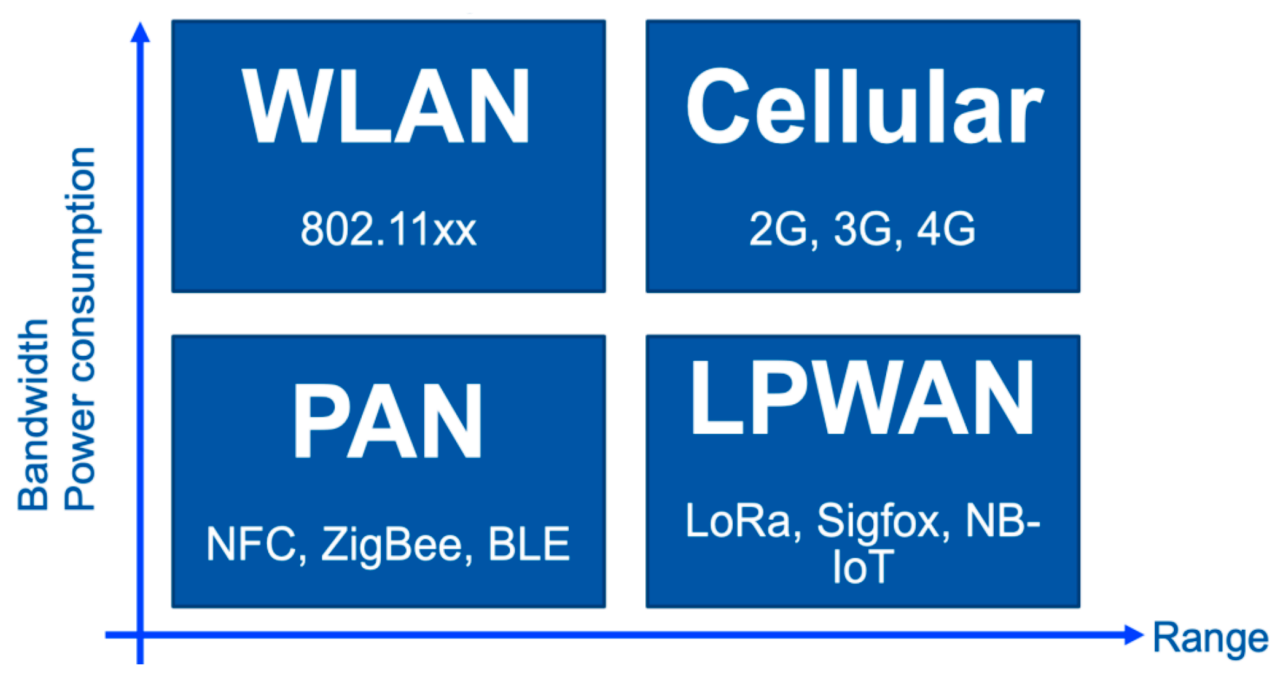

Figure 1. Wireless networks

When not connected to the electrical grid, there is a trade-off between the range over which the device can communicate, its maximum data rate and its power consumption. If there is power available or the batteries can be recharged often, the device can afford to have higher data throughput and/or move further from the source.

Personal Area Networks (PAN) offer you a solution if your device is only a few centimeters/meters away. Bluetooth printers or contactless cards using RFID (RadioFrequency Identification) or NFC (Near-Field Communication) are just some PAN examples.

Wireless Local Area Networks (WLAN) and cellular networks provide you high throughput at longer ranges.

Low-Power Wide-Area Network (LPWAN) focuses on low-cost devices that need to operate on batteries for long periods of time and send small volumes of data. 


\subsection{CERN wireless loT networking}

CERN users have been connecting all kind of devices for several years via Ethernet, Wi-Fi, cellular networks or even home-made solutions. However, they were lacking the capabilities of LPWAN networks to easily deploy battery-powered sensors at any location and at an affordable price.

CERN IT department decided to offer a complete and centralized LPWAN service to avoid uncontrolled, unmanaged and competing networks installed by the different services requiring this kind of solution.

\subsection{CERN LPWAN Solution study}

LPWAN offers wireless connectivity for large areas and complements other technologies such as cellular machine-to-machine (M2M), Wi-Fi or personal area networks (PAN).

An LPWAN provides long range (up to several kilometers) communication capability to low power consumption devices (up to several years without changing the battery) in a costeffective way (low-device cost). The price to pay is the low bit rate (few kbps).

An LPWAN is the perfect match for any kind of sensor or meters sending small amounts of data several times per day. These networks are conceived to support a massive number of devices since they spend most of their time silent.

\subsubsection{Technology choice}

Different solutions in the market were assessed, including LoRaWAN ${ }^{[2]}$, NB-IoT $^{[3]}$, SigFox ${ }^{[4]}$, Weightless-P ${ }^{[5]}$, Weightless-N ${ }^{[5]}$, Ingenu ${ }^{[6]}$, SymphonyLink ${ }^{[7]}$, QoWisio ${ }^{[8]}$, Neul ${ }^{[9]}$ or Waviot ${ }^{[10]}$.

Some of the requirements the study was based on were:

- The backend access and provisioning of the solution should be flexible enough to integrate them with other CERN services.

- Sub-GHz bands as the propagation losses in the underground facilities increase dramatically for frequencies over $1 \mathrm{GHz}$.

- Good radio properties so the sensors could be deployed both outdoors and indoors without having to add any distribution antenna system within the buildings.

- Data source identity, integrity checking and encryption.

- Data privacy and storage ownership.

- Secured communication between backend, gateways and user applications.

- Standards compliant solution with extended technical documentation to provide support and maintenance for the solution.

- Reliability and scalability of the solution.

- Technology maturity and adoption.

- Device and chips availability.

- A minimum set of Quality of Service (QoS) mechanism such as forward error correction and acknowledgment option.

- Easiness to deploy and use.

- CAPEX and OPEX costs.

Being at the border of two countries, national operator solutions were not considered so a self-contained solution deployed on premises was preferred. Therefore, the solution should work on the unlicensed Industrial, Scientific and Medical (ISM) band. 
LoRaWAN was chosen because of its maturity, good radio and capacity properties, availability of devices and chips, backend features and its facility of integration with other services.

LoRa $^{[11]}$ (Long Range) is used as the radio modulation in the physical layer while the LoRaWAN protocol is used for the MAC layer.

\subsubsection{Business model choice}

Both commercial and open-source as well as private and self-operated solutions were studied. Following the department strategy for services provisioning, and after assessing some of the solutions according to the requirements previously mentioned; an open-source based service hosted on premises was favoured.

A new set of requirements was added to the list to select the right open-source solution: - Compliance with LoRaWAN 1.1 specification.

- Adaptative Data Rate support.

- OTTA and ABP support.

- Backend monitoring and management APIs for integration into CERN services.

- Backend communication protocols.

- High availability and scalability.

- Support for downlink messages.

The Things Network (TTN) ${ }^{[12]}$ was selected as the backend.

\section{LoRaWAN at CERN}

\subsection{Service}

A simplified version of LoRaWAN architecture is shown on the next figure:

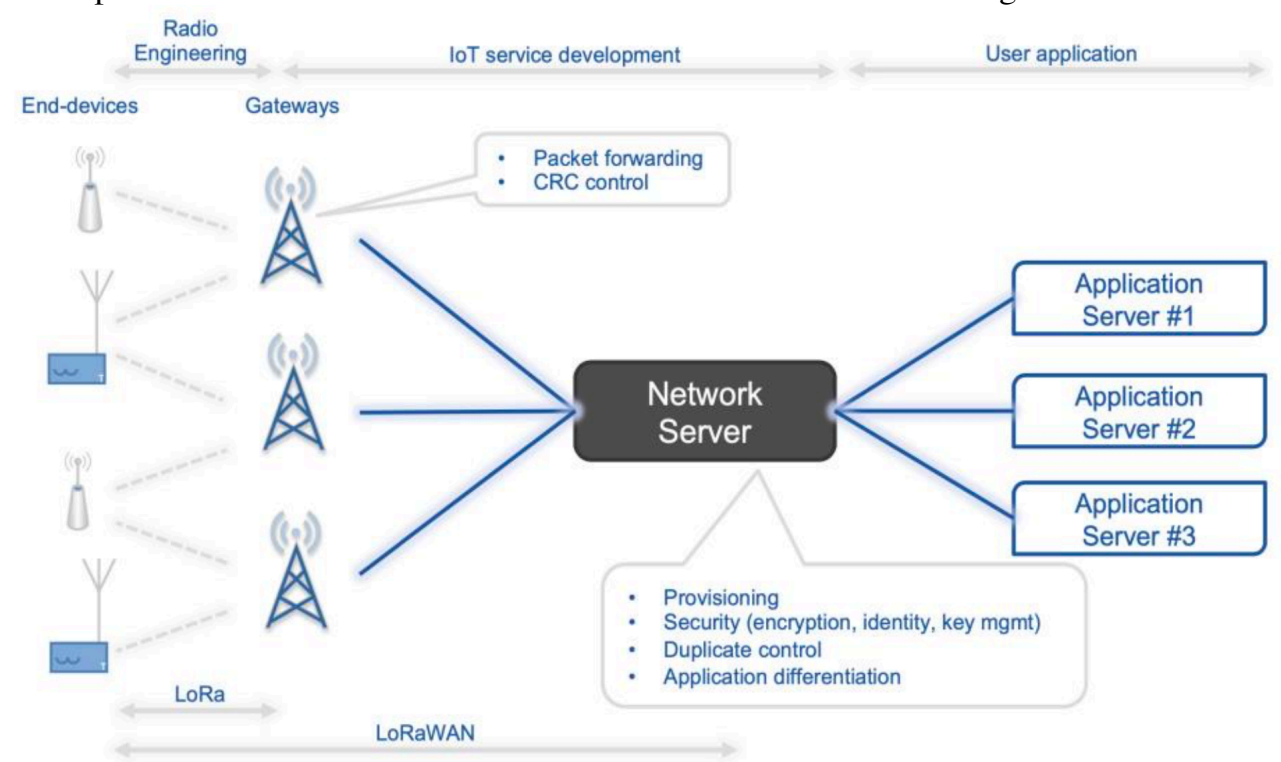

Figure 2. LoRaWAN simplified architecture to:

In order to integrate the network server into CERN current services, it had been adapted 
- Deploy the solution on premises using the CERN's cloud standard tools for agile development, high availability and orchestration.

- Integrate the gateways into CERN's SNMP monitoring service (Spectrum in figure 3).

- Integrate the different solution elements into the department monitoring service via ElasticSearch (AI-Monitoring in figure 3).

- Integrate user identity, devices and application provisioning as well as encryption keys handling into CERN's standard network tools (both for administration and user interfaces). Integrate the data consumption and analysis into standard IT tools via Kafka.

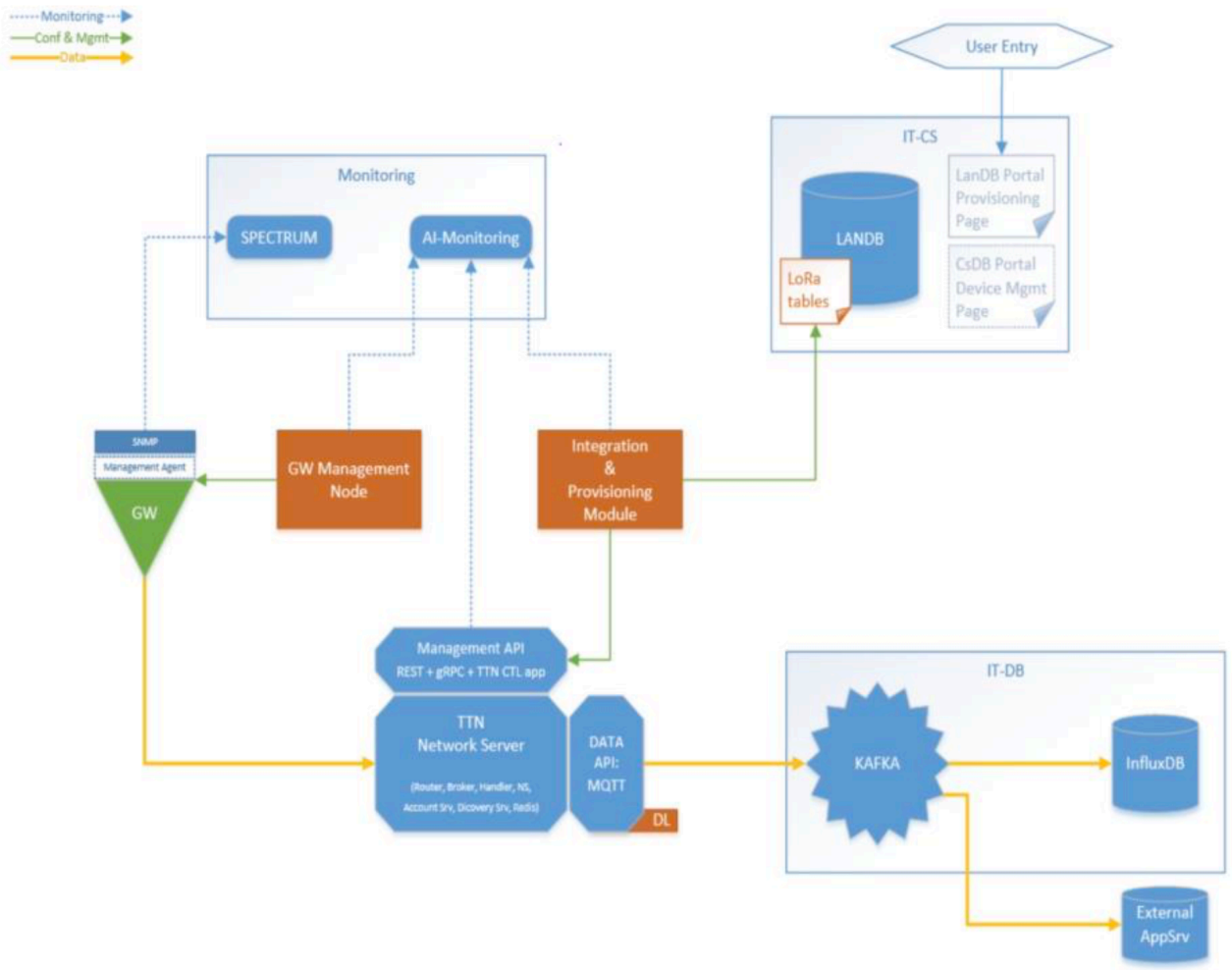

Figure 3. CERN LoRaWAN architecture

An end-user only needs to follow four steps to have his sensors and application running: - Declare the application and user access lists via the already known standard IT web tools. Declare the devices via the same tools and upload the encryption keys into them.

- Define in Kafka the function to decode the data and the database/scheme where the data will be stored.

- Consume the data via his own developed/purchased application or use one of the standard IT data services to analyse it.

\subsection{Radio coverage}

Outdoor coverage is assured campus-wide thanks to 11 gateways installed in the different sites. As different gateways overlap in different areas, the user can profit from some spatial redundancy in case one of the gateways fails.

CERN EN department is hardening the LoRaWAN chip so it can work under radiation. As the sensors become radiation tolerant, the network can be extended to the underground 
facilities of CERN; opening a new set of use cases. The first tests in the LHC have proven to be very positive.

\section{Conclusions}

CERN has deployed a Low Power Wireless Area Network (LPWAN) to complement the existing wired and wireless networks and the required service infrastructure to manage the provisioning, orchestration, data transfer, security and operation for the connected devices.

A study led to the deployment of a private LoRaWAN network based on an open-source solution (The Things Network) integrated into existing standard IT services.

A user can use the already known IT tools to define the application, provision the devices and consume and analyse the data.

\section{Future work}

The deployment of LoRaWAN in the underground facilities will allow to easily deploy battery-power sensors at any location at low cost.

New requirements and use cases will soon appear as well as other technologies and standards. IT would need to follow up the IoT evolution to cope with the organisation needs.

\section{References}

1. Alliance for Internet of Things Innovation (AIOTI), http://aioti.eu

2. LoRa Alliance, https://lora-alliance.org/

3. GSMA NB-IoT, https://www.gsma.com/iot/narrow-band-internet-of-things-nb-iot/

4. SigFox, https://www.sigfox.com/en

5. Weightless Specification, http://www.weightless.org

6. Ingenu, https://www.ingenu.com/

7. Link Labs Synphony Link ${ }^{\mathrm{TM}}$, https://www.link-labs.com/symphony/

8. QoWisio, https://www.qowisio.com/en//

9. Neul, https://www.neul.com/

10. WAVIoT, https://waviot.com/

11. LoRa, https://www.semtech.com/lora/what-is-lora

12. The Things Network, https://www.thethingsnetwork.org/

13. Tsisaris V, Karnouskos S, Höller J, Boyle D, Mulligan C, Intenet of Things (Academic Press, 2019) 